\title{
Regiones de economía abierta y cerrada*
}

\section{Antonio Daher* *}

Este estudio identifica las regiones productoras de bienes, de exportación y domésticas de Chile, precisando los distintos grados de apertura de las economías regionales y su desigual contribución al comercio exterior luego de una década y media de implementación del nuevo modelo de desarrollo.

Esta "especialización" regional es contrastada con la orientación territorial divergente de las inversiones en los sectores productores de bienes y los de servicios, demostrándose que estos últimos, y más aún las exportaciones, se constituyen en importantes factores de diferenciación -si no de discriminación-entre las regiones.

La especialización exportadora o doméstica acusa una muy distinta vulnerabilidad regional frente a las fluctuaciones de los mercados locales y mundiales, y ante los impactos de las políticas macroeconómicas y de comercio exterior.

\section{Introducción}

En el marco de la globalización destaca la experiencia chilena como precursora entre los países no industrializados de un modelo de desarrollo basado en una economía abierta y en el fomento de las exportaciones y, en tanto tal, indisociable del comercio exterior.

En este contexto es de gran relevancia conocer el comportamiento de los sectores productores de bienes y los de servicios, las variaciones en su despliegue territorial, y la participación diferencial de cada región del país en las exportaciones.

En Chile, entre 1940 y 1990, los sectores productores de bienes -agricultura, pesca, minería e industria- representaron como máximo 47 y $47.2 \%$ del producto geográfico bruto (PGB) en 1955 y 1974 respectivamente, y como mínimo 33.9\% en 1981 (Behrens, 1990: A 32-34). Desde 1977 en adelante, es decir, durante el periodo de mayor apertura económica, los sectores productores de bienes nunca lograron llegar a $40 \%$ del producto. Por el contrario, su participación es la menor en los últimos cincuenta años, con-

* Una versión preliminar de este estudio fue expuesta en el Seminario Internacional Integración Latinoamericana y Territorio, en la Universidad de Buenos Aires. La investigación se ha realizado con el patrocinio del Fondo Nacional de Ciencia y Tecnología de Chile.

* El autor es académico de la Pontificia Universidad Católica de Chile, Instituto de Estudios Urbanos. 
firmándose el incremento relativo de los sectores productores de servicios y la tendencia terciarizadora. En efecto, entre 1985 y 1990 , durante el mayor auge exportador, los sectores productores de bienes crecieron en $29.2 \%$ mientras que los sectores de servicios lo hicieron, sorprendentemente, en $38.3 \%$ (Banco Central de Chile, 1991: 1575).

Las exportaciones, a su vez, fueron equivalentes a $11.7 \%$ del PGB en 1970 , a $20.9 \%$ en 1980 (De la Cuadra, 1988: 34) y a $27.5 \%$ en 1990 (véase el cuadro 3) mostrando una tendencia inversa a la del sector productor de bienes, del cual ciertamente son un subconjunto.

La suma de exportaciones e importaciones prácticamente se duplica entre 1986 y 1990, pasando de 7132 millones de dólares a 15260 millones de dólares (Servicio Nacional de Aduanas, 1990). Esta última cifra indica que el comercio exterior ha llegado a representar prácticamente la mitad del producto nacional.

Pero tanto o más importante que el incremento absoluto del comercio exterior es la propia diversificación del sector exportador y el cambio en la composición de las importaciones. En efecto, mientras que en 1970 los embarques mineros aportaban $74 \%$ del valor exportado, los de la industria $20.1 \%$ y los agropecuarios 5.9\% (De la Cuadra, 1988: 33), en 1989 la minería contribuía sólo con $55.4 \%$, la industria lo hacía con un importante $32.3 \%$, y los productores agropecuarios, forestales y del mar con $12.3 \%$ (Banco Central de Chile, 1991: 1538 y 1 540). Esta distribución distinta y su variada composición intrasectorial permiten prefigurar una geografía de las exportaciones radicalmente diferente, a la vez que una fuerte transformación en la contribución regional al comercio exterior.

Por otra parte, las importaciones acusan también cambios significativos en su destino sectorial: los bienes de consumo bajan de $30 \%$ en 1981 a $11.8 \%$ en 1990 ; los intermedios pasan de 50.4 a $57.6 \%$ y los de capital suben de 19.6 a $30.3 \%$ en los mismos años (Banco Central de Chile, 1983: 1 704; 1990: 2 506-2 507). Esta nueva composición de las importaciones también permite inferir una modificación en su orientación geográfica, considerando que el destino de los bienes de consumo puede presumirse análogo a la distribución demográfica y de ingresos, mientras que la localización para los bienes intermedios y de capital se asimilaría más a la geografía de las actividades económicas en general.

Adicionalmente, el incremento del comercio exterior hizo crecer entre 1981 y 1989 de 26.9 a $39.1 \%$ la participación de los gravámenes aduaneros en los ingresos generales de la nación (Servicio Nacional de Aduanas, 1990: 16), lo que a su vez se tra- 
dujo en una menor contribución de la región metropolitana a tales ingresos, bajando de $55.3 \%$ en 1981 (y $66.7 \%$ en 1982) a sólo $37.1 \%$ en 1989 (cifras de la Tesorería General de la República referidas por la Secretaría Regional Ministerial de Economía, Fomento y Reconstrucción de Valparaíso).

Así pues, constatada la profunda relación entre los procesos económicos y las transformaciones territoriales en Chile en los últimos años (Daher, 1990a), no puede sino reconocerse que en importante medida aquéllos y éstas encuentran en la economía abierta y en el desarrollo exportador su factor más decisivo.

Los objetivos de este estudio son, por lo mismo, definir las regiones productoras de bienes y las de servicios de Chile, a partir de los cambios en la localización de tales sectores; analizar la contribución de cada región a las exportaciones nacionales, para identificar las regiones de exportación y las orientadas al mercado doméstico, y relacionar finalmente los resultados anteriores con la distribución regional de las inversiones proyectadas, previendo así tendencias de mediano plazo.

En síntesis, el propósito central de la investigación que se expone es precisar los distintos grados de apertura de las economías regionales en Chile, luego de una década y media de implantación del nuevo modelo. Con ello será posible conocer la desigual sensibilidad de cada región a la variación de los precios y las demandas internacionales, y prever el efecto diferencial en las distintas regiones de los cambios en los precios relativos entre los bienes y los servicios. El impacto territorial -y social- de las políticas cambiaria, arancelaria y en general de comercio exterior (Daher, 1990b) podrá ser anticipado-especificado- y eventualmente manejado en cada una de las regiones.

En 1992, la caída del tipo de cambio real y de los precios internacionales de los principales productos de exportación, a la vez que la mayor probabilidad de acuerdos de integración y libre comercio, hacen tanto más necesario precisar las regiones de economía abierta y las de economía cerrada, para la evaluación territorial de los efectos negativos y positivos de tales procesos.

\section{Regiones productoras de bienes, de exportación y domésticas}

La participación de los sectores productores de bienes en el PGB de cada región indica el grado de productividad de las respectivas economías. El cuadro 1 permite observar que, aun cuando para el conjunto del país los sectores productores de bienes se aproximaron a $40 \%$ del PGB nacional tanto en 1970 como 1990 -con una 
pronunciada baja relativa en los años ochenta- la situación de las regiones, en cambio, presenta variaciones de gran magnitud.

\section{CUADRO 1}

PGB Regional de bienes: participación en el PGB Regional y contribución al PGB Nacional de bienes, Chile, 1970 y 1990

(Millones de pesos de 1977)

\begin{tabular}{|c|c|c|c|c|c|c|}
\hline Región & $\begin{array}{c}P G B \text { de } \\
\text { bienes } \\
\text { regional } \\
1970(\mathrm{~A})\end{array}$ & $\begin{array}{l}(A) \\
P G B \\
\text { Reg. }\end{array}$ & $\begin{array}{c}(A) \\
P G B \text { de } \\
\text { bienes } \\
(B)\end{array}$ & $\begin{array}{c}\text { PGB de } \\
\text { bienes } \\
\text { regional } \\
1990\end{array}$ & $\begin{array}{c}(B) \\
P G B \\
\text { Reg. }\end{array}$ & $\begin{array}{c}(B) \\
\text { PGB de } \\
\text { bienes }\end{array}$ \\
\hline I & 2914 & 42.9 & 2.6 & 9835 & 51.0 & 5.2 \\
\hline II & 6507 & 56.3 & 5.8 & 17607 & 61.6 & 9.2 \\
\hline III & 2656 & 51.1 & 2.4 & 5728 & 52.1 & 3.0 \\
\hline IV & 1542 & 35.1 & 1.4 & 5174 & 49.3 & 2.7 \\
\hline V & 12495 & 41.6 & 11.1 & 18281 & 39.5 & 9.6 \\
\hline VI & 8887 & 66.9 & 7.9 & 17874 & 66.3 & 9.4 \\
\hline VII & 4550 & 46.9 & 4.0 & 7832 & 38.2 & 4.1 \\
\hline VIII & 13745 & 51.3 & 12.2 & 26623 & 49.7 & 14.0 \\
\hline IX & 3748 & 45.7 & 3.3 & 6361 & 43.4 & 3.3 \\
\hline $\mathrm{X}$ & 5235 & 42.6 & 4.6 & 9719 & 43.2 & 5.1 \\
\hline XI & 338 & 27.5 & 0.3 & 806 & 33.4 & 0.4 \\
\hline XII & 4538 & 62.5 & 4.0 & 10004 & 57.9 & 5.2 \\
\hline $\mathrm{RM}$ & 45625 & 35.0 & 40.4 & 54958 & 27.2 & 28.8 \\
\hline País & 112780 & 39.8 & 100.0 & 190802 & 39.7 & 100.0 \\
\hline
\end{tabular}

Fuentes: (A) ODEPLAN (1990a). (B) Proyección del autor.

De hecho, si bien en ambas fechas, la VI región -minero-agrícola- aparece como la más productiva (dos tercios de su economía regional), la menos productiva en 1970, la XI, en cambio, cede su lugar en 1990 a la región metropolitana (RM), la que presenta la economía más cerrada del país. Esta región capital baja su producción de bienes de 35 a $27.2 \%$, y de 40.4 a $28.8 \%$ su contribución al PGB nacional de bienes. A pesar de todo, por su volumen económico, la RM es la que mayor participación tiene en la producción de bienes del país.

Otras regiones de alta productividad son la II y la XII, minera y minero-energética respectivamente. En el otro extremo, en 1970 sólo estaban bajo el promedio nacional las regiones XI y IV, amén de la metropolitana, y en 1990 la XI, VII, V y RM; es decir, una región de frontera de desarrollo, otra especializada en generación de energía, y las dos principales sedes metropolitanas. 
En el transcurso de las dos décadas, incrementaron notoriamente su productividad las regiones IV (de 35.1 a $49.3 \%$ ) y I (de 42.9 a $51 \%$ ), aquella minero-agrícola y ésta minero-pesquera, sede de la mayor zona franca. Los decrementos más radicales corresponden a la RM y la VII región (esta última de 46.9 a 38.2 por ciento).

En la contribución a la producción nacional de bienes, destacan positivamente las regiones I (2.6 a $5.2 \%$ ), IV (1.4 a $2.7 \%$ ) y II (5.8 a $9.2 \%$ ). Negativamente sólo las regiones $\mathrm{V}$ y metropolitana. Fuera de éstas dos, todas las demás incrementan o al menos conservan su proporción nacional.

En el cuadro 2 se muestra la distribución regional del comercio exterior a través de la participación de cada región en las exportaciones y -con un sesgo portuario- en las importaciones. Éstas acusan una marcada concentración en la V región (sobre 60\%) y en la VIII, segundo frente portuario del país. Es importante también la proporción de la RM y el porcentaje conjunto de las dos primeras regiones. Con todo, si se suman los valores de las regiones V y metropolitana -geográficamente contiguas y demográficamente concentradas- se supera a $70 \%$ de las importaciones nacionales.

\section{CUADRO 2}

Exportaciones por región de origen e importaciones por aduanas regionales. Chile, 1990

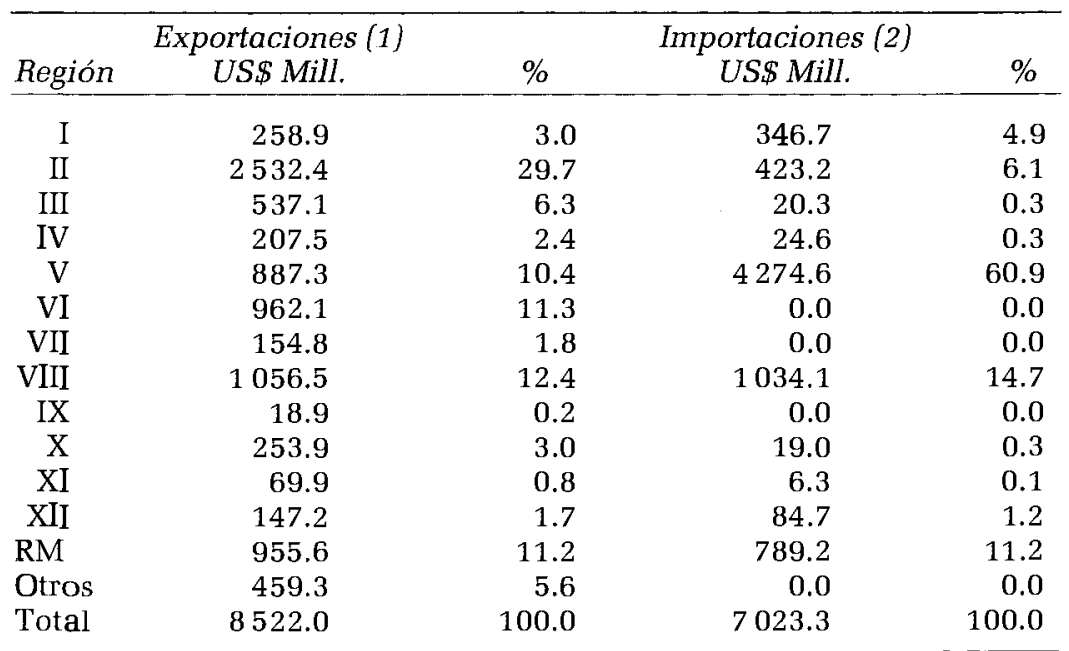

(1) Valores FOB.

(2) Declaración de importaciones.

Fuente: Servicio Nacional de Aduanas (1990). 
Las exportaciones en cambio, muestran un patrón nítidamente más disperso. La región más exportadora es la II, cuya minería aporta cerca de $30 \%$ de las divisas por ventas al exterior. A continuación siguen las regiones VIII (forestal-industrial), VI, RM y V, con porcentajes que fluctúan entre 12.4 y $11.2 \%$. Estas cinco regiones contribuyen con tres cuartas partes del total exportado por el país en 1990.

En contraste, la región menos exportadora es la IX, con sólo $0.2 \%$ del total nacional. A ella se agregan la XI, XII y VIL Estas cuatro regiones -dos energéticas y dos con el mayor índice de pobreza extrema relativa- contribuyen sólo con $4.5 \%$ de las exportaciones totales.

El cuadro 3 expone, junto a la contribución de cada región al PGB nacional en 1990, la proporción exportada de ese producto por cada una de ellas. Este último indicador muestra la relevancia del sector exportador en el interior de las economías regionales, y por lo mismo permite discernir las regiones más "especializadas" en exportar. Estas, a su vez, serían proporcionalmente las más sensibles a las fluctuaciones en los mercados externos y a las políticas de comercio exterior del propio país.

CUADRO 3

PGB por región y exportaciones como proporción del PGB regional, Chile, 1990

\begin{tabular}{crrr}
\hline & $P G B(1)$ & & Exportaciones (2) \\
Región & US\$ Mill & $\%$ & $P G B$ \\
\hline I & 1244.9 & 4.0 & 20.8 \\
II & 1842.9 & 5.9 & 137.4 \\
III & 709.3 & 2.3 & 75.7 \\
IV & 677.8 & 2.2 & 30.6 \\
V & 2982.9 & 9.6 & 29.7 \\
VI & 1739.8 & 5.6 & 55.3 \\
VII & 1323.5 & 4.3 & 12.0 \\
VIII & 3454.2 & 11.1 & 30.6 \\
IX & 947.3 & 3.1 & 2.0 \\
X & 1453.2 & 4.7 & 17.5 \\
XI & 155.6 & 0.5 & 44.9 \\
XII & 1114.6 & 3.6 & 13.2 \\
RM & 13047.4 & 42.1 & 7.3 \\
Otros & 299.3 & 1.0 & 160.1 \\
Total & 30992.7 & 100.0 & 27.5 \\
\hline
\end{tabular}

Fuentes: (1) Proyección del autor; (2) Cuadro 2. 
Este tercer cuadro permite observar que seis regiones están menos especializadas en exportar que el país en su conjunto: bajo $27.5 \%$ nacional están la I -probablemente subvalorada por la II-, X, la VII, la XII, la RM y la IX. Llama la atención el exiguo $7.3 \%$ de la metropolitana.

Al revés, entre las más especializadas en exportar destaca la región II, obviamente sobrevalorada al exceder sus ventas externas al propio producto regional, y las regiones III y VI, todas de gran relevancia minera, amén de la importancia agrícola de esta última.

Las regiones con más aporte a las exportaciones nacionales que al PGB del país son la II, III, IV, VI, VIII y XI. Las cinco regiones señaladas más arriba con mayor contribución a las exportaciones nacionales (75\%), generan $74.3 \%$ del PGB. Sin embargo, excluida la región metropolitana, el porcentaje de exportaciones cae sólo a $63.8 \%$, mientras que la participación en el PGB nacional baja radicalmente a menos de un tercio.

Las cuatro regiones del norte del país, eminentemente mineras, suman $41.4 \%$ del valor exportado y sólo $14.4 \%$ del PGB nacional. En contraste, las tres sedes metropolitanas (RM, V y VIII), con casi $63 \%$ del producto, generan solamente $34 \%$ de las ventas externas.

El cuadro 4 muestra el producto y las exportaciones regionales per cápita. En cuanto al primero, el valor máximo corresponde a la XII región -escasamente poblada y única productora de petróleo-cuyos 6971 dólares contrastan con los 1190 de la IX. Las tres regiones señaladas, a través de indicadores de vivienda, como las de mayor índice de pobreza extrema relativa del país, es decir la IV, IX y VII, son las que a su vez presentan los menores valores de producto per cápita, y son las mismas que detentan muy bajos índices de exportación por habitante.

Las regiones con más alto producto per cápita son, en orden descendente, la XII, la II, la III, la I y la VI. En contraste, bajo el promedio nacional se ubican todas las regiones centrales del país, de la IV a la XI, con excepción de la VI y de la metropolitana, muy cercana ésta, por su alta ponderación, al promedio nacional.

A su vez, las exportaciones por habitante fluctúan entre un máximo de 6508 dólares de la región II y un mínimo de apenas 23 dólares en la IX. Además de la II, presentan valores altos la tercera y sexta regiones. Bajo el promedio nacional de 646 dólares se encuentran todas las regiones centrales del país, la IV a la X, con la sola excepción de la VI. Una vez más, las regiones extremas menos pobladas destacan en los indicadores por habitante. 
CUADRO 4

PGB y exportaciones regionales per cápita, Chile, 1990

\begin{tabular}{ccc}
\hline Región & $\begin{array}{c}\text { Producto (1) } \\
\text { per cápita (3) } \\
\text { US\$ }\end{array}$ & $\begin{array}{c}\text { Exportaciones (2) } \\
\text { per cápita (3) } \\
\text { US\$ }\end{array}$ \\
\hline I & 3476 & 720 \\
II & 4731 & 6508 \\
III & 3585 & 2725 \\
IV & 1393 & 425 \\
V & 2158 & 642 \\
VI & 2767 & 1482 \\
VII & 1575 & 183 \\
VIII & 2063 & 630 \\
IX & 1190 & 23 \\
X & 1575 & 274 \\
XI & 1938 & 873 \\
XII & 6971 & 924 \\
RM & 2492 & 182 \\
Total & 2353 & 646 \\
\hline
\end{tabular}

Fuentes: (1) Proyección del autor; (2) cuadro 2; (3) Población proyectada para 1990. ODEPLAN (1990b).

Los cuadros 5 y 6 hacen posible definir las regiones productoras de bienes, de exportación y domésticas de Chile, a la vez que permiten un análisis comparado de la productividad y extroversión de cada una, y por diferencia, de la orientación al mercado local. Adicionalmente, ambos cuadros contrastan la contribución regional a la producción nacional de bienes y a las exportaciones totales del país.

El análisis comparado lleva a la identificación de una cierta tipología de regiones. Un primer tipo corresponde a los casos de alta productividad y alta extroversión -y por ende baja orientación al mercado doméstico- a la vez que de mediana o alta contribución a los totales nacionales de exportaciones y producción de bienes.

Como se aprecia en el cuadro 6, a este tipo corresponden las regiones II, VI y III, esta última con un menor aporte nacional al sector productor de bienes. Ciertamente estas son las regiones de economía netamente exportadora del país.

La región típicamente doméstica, en el otro extremo, es la VII: muy bajas son su producción de bienes y extroversión y menor su contribución relativa al PGB de bienes y al valor exportado nacionales. La región metropolitana es, salvo por su enorme ponderación en la economía del país, tanto y más cerrada. Con todo, su participación, del orden de $40 \%$ en el producto nacional, la hace 
CUADRO 5

Regiones productoras de bienes, de exportación y domésticas, Chile, 1990

\begin{tabular}{cccccc}
\hline & $\begin{array}{c}\text { Productividad } \\
(A)\end{array}$ & $\begin{array}{c}\text { Extroversión } \\
(B)\end{array}$ & & $\begin{array}{c}\text { PGBR de } \\
\text { Bienes }\end{array}$ & $\begin{array}{c}\text { Export. } \\
\text { Regional } \\
\text { Export. }\end{array}$ \\
Región & $\begin{array}{c}\text { PGBR de bienes } \\
\text { Export. Reg. }\end{array}$ & $\begin{array}{c}\text { Introversión } \\
\text { PGBR }\end{array}$ & $\begin{array}{c}\text { PGBN de } \\
(A-B)\end{array}$ & $\begin{array}{c}\text { PGienes } \\
\text { Nacional }\end{array}$ \\
\hline I & 51.0 & 20.8 & 30.2 & 5.2 & 3.0 \\
II & 61.6 & 137.4 & -75.8 & 9.2 & 29.7 \\
III & 52.1 & 75.7 & -23.6 & 3.0 & 6.3 \\
IV & 49.3 & 30.6 & 18.7 & 2.7 & 2.4 \\
V & 39.5 & 29.7 & 9.8 & 9.6 & 10.4 \\
VI & 66.3 & 55.3 & 11.0 & 9.4 & 11.3 \\
VII & 38.2 & 12.0 & 26.2 & 4.1 & 1.8 \\
VIII & 49.7 & 30.6 & 19.1 & 14.0 & 12.4 \\
IX & 43.4 & 2.0 & 41.4 & 3.3 & 0.2 \\
X & 43.2 & 17.5 & 25.7 & 5.1 & 3.0 \\
XI & 33.4 & 44.9 & -11.5 & 0.4 & 0.8 \\
XII & 57.9 & 13.2 & 44.7 & 5.2 & 1.7 \\
RM & 27.2 & 7.3 & 19.9 & 28.8 & 11.2 \\
No. Reg. & - & - & - & - & 5.6 \\
País & 39.7 & 27.5 & 12.2 & 100.0 & 100.0 \\
\hline
\end{tabular}

Fuentes: Cuadros 1, 2 y 3.

liderar en el ranking de producción de bienes, y en menor medida, en el de exportaciones totales.

Un tercer tipo es el de las regiones VIII, IV, X y V, todas ellas de productividad y extroversión medianas y, en consecuencia, con una orientación también mediana al mercado local. Pero en su aporte relativo a los bienes producidos y exportados del país muestran conductas diversas, también asociadas a la diferencial de tamaño de sus economías regionales. Así, mientras la VIII y la V destacan positivamente en el nivel nacional en tales aportes, la IV y la $\mathrm{X}$ se ubican en un rango medio-bajo.

Las regiones XII, IX, XI y I conforman un cuarto grupo, esta vez más propiamente "atípico". Aunque, como se ha advertido, la región I queda distorsionada, las otras tres corresponden a alta (XII), media (IX) y baja (XI) productividades. La XII es paradójicamente la más doméstica del país (que, como se sabe, está lejos de autoabastecerse de hidrocarburos); mientras la XI, por oposición, es de las más extrovertidas, y la IX, la más introvertida.

Finalmente, el cuadro 7 relaciona la contribución regional al PGB de bienes y a las exportaciones nacionales, por una parte, y 
CUADRO 6

Ranking de regiones productoras de bienes, de exportación y doméstica, Chile, 1990

\begin{tabular}{|c|c|c|c|c|c|}
\hline Rankin & $\begin{array}{l}\text { Regiones pro- } \\
\text { g duct. de bienes }\end{array}$ & $\begin{array}{l}\text { Regiones de } \\
\text { Export. }\end{array}$ & $\begin{array}{l}\text { Regiones } \\
\text { Domést. }\end{array}$ & $\begin{array}{l}\% \text { de Bienes } \\
\text { Nacionales }\end{array}$ & $\begin{array}{l}\% \text { Export. } \\
\text { Nacionales }\end{array}$ \\
\hline 10. & VI & II & XII & RM & II \\
\hline 20. & II & III & IX & VIII & VIII \\
\hline 30. & XII & VI & I & V & VI \\
\hline 40. & III & XI & VII & VI & RM \\
\hline 50. & I & VIII & $\mathrm{X}$ & II & V \\
\hline 60. & VIII & IV & $\mathrm{RM}$ & XII & III \\
\hline 70. & IV & $\begin{array}{c}\mathrm{V} \\
\text { Media país }\end{array}$ & VIII & I & I \\
\hline 80. & IX & I & $\begin{array}{c}\text { IV } \\
\text { Media país }\end{array}$ & $\mathrm{X}$ & $\mathrm{X}$ \\
\hline 9o. & $\begin{array}{r}\mathrm{X} \\
\text { Media país }\end{array}$ & $\mathrm{X}$ & VI & VII & IV \\
\hline $10 o$ & $\mathrm{~V}$ & XII & V & IX & VII \\
\hline 110. & VII & VII & XI & III & XII \\
\hline 120. & XI & $\mathrm{RM}$ & III & IV & XI \\
\hline 130. & RM & IX & II & XI & IX \\
\hline
\end{tabular}

Fuente: Cuadro 5.

por otra las inversiones proyectadas en los sectores de producción de bienes y de servicios desagregadamente.

En términos globales se constata que la inversión proyectada en el sector productor de bienes supera a la propia del sector de servicios y ello aún considerando que las inversiones en agricultura y pesca están escasamente registradas (Aninat, 1990). Esto es importante teniendo en cuenta que, como se señalara antes, los servicios tuvieron una tasa de crecimiento muy superior en los últimos años.

Ahora bien, la inversión proyectada en el área productora de bienes muestra una cierta dispersión relativa entre las regiones pero, aunque claramente se ha roto el patrón monopolar, con una fuerte concentración en las II (una cuarta parte del total), X y VIII. Estas tres regiones captan más de la mitad de las inversiones sectoriales, con $56.5 \%$. Ellas mismas suman $45.1 \%$ de las exportaciones del país.

Por el contrario, otras cuatro regiones, la IV, la V, la VII y la XI, apenas detentan $3.6 \%$ de los proyectos del sector productor de bienes, a pesar de contribuir en conjunto con $16.8 \%$, con $15.4 \%$ a las exportaciones nacionales. 
CUADRO 7

Inversión proyectada ${ }^{1}$ en los sectores productores de bienes y de servicios, por región, Chile, 1990-1995

\begin{tabular}{|c|c|c|c|c|c|c|}
\hline Región & $\begin{array}{l}\text { Inversión } \\
\text { Sector prod. } \\
\text { de bienes } \\
\text { US\$ Mill. }\end{array}$ & . & $\begin{array}{l}\text { Inversión } \\
\text { Sector de } \\
\text { servicios } \\
\text { US\$ Mill. }\end{array}$ & $\%$ & $\begin{array}{c}\% \\
\text { Exportaciones } \\
\text { nacionales }\end{array}$ & $\begin{array}{c}\% \\
\text { Prod. de } \\
s \quad \text { bienes } \\
\text { nacionales }\end{array}$ \\
\hline I & 581.4 & 5.5 & 137.2 & 1.5 & 3.0 & 5.2 \\
\hline II & 2717.6 & 25.6 & 26.2 & 0.3 & 29.7 & 9.2 \\
\hline III & 898.6 & 8.5 & 381.4 & 4.2 & 6.3 & 3.0 \\
\hline IV & 109.8 & 1.0 & 8.0 & 0.1 & 2.4 & 2.7 \\
\hline V & 142.0 & 1.3 & 497.2 & 5.5 & 10.4 & 9.6 \\
\hline VI & 538.5 & 5.1 & 327.3 & 3.6 & 11.3 & 9.4 \\
\hline VII & 75.0 & 0.7 & 759.8 & 8.4 & 1.8 & 4.1 \\
\hline VIII & 1334.7 & 12.6 & 1624.7 & 17.9 & 12.4 & 14.0 \\
\hline IX & 669.5 & 6.3 & 34.6 & 0.4 & 0.2 & 3.3 \\
\hline $\mathrm{X}$ & 1944.2 & 18.3 & 948.3 & 10.5 & 3.0 & 5.1 \\
\hline $\mathrm{XI}$ & 66.7 & 0.6 & 3.9 & 0.0 & 0.8 & 0.4 \\
\hline XII & 535.0 & 5.0 & 122.8 & 1.4 & 1.7 & 5.2 \\
\hline RM & 568.5 & 5.3 & 1794.7 & 19.8 & 11.2 & 28.8 \\
\hline No. Reg & 451.8 & 4.2 & 2391.8 & 26.4 & 5.6 & - \\
\hline País & 10633.3 & 100.0 & 9057.9 & 100.0 & 100.0 & 100.0 \\
\hline
\end{tabular}

1 Inversión proyectada, empresas públicas y privadas, 1990-1995 (Aninat, 1990). Fuentes: Cuadros 1 y 2.

Además, es notoria la baja captación -en proporción a su contribución a la producción de bienes y a las exportaciones- de las regiones VI y metropolitana. Esta última percibiría sólo $5.3 \%$ de las inversiones, generando $11.2 \%$ del valor exportado y $28.8 \%$ del producto de bienes. La VI región capta un porcentaje cercano sólo a la mitad de lo que aporta en ambos rubros.

Las regiones IX y $\mathrm{X}$, en cambio, acusan una muy favorable percepción de recursos: casi una cuarta parte del total de inversiones del sector productor de bienes, contra $8.4 \%$ del producto respectivo y sólo $3.2 \%$ de las exportaciones. Particularmente significativa es la inversión orientada a la IX región, en el área industrial-forestal, considerando el impacto que aquélla podría tener en los indicadores de pobreza locales.

En el sector de servicios, la orientación nacional de las inversiones sigue un patrón también de dispersión polarizada, pero de contenido diferente. Esta vez, con la excepción de las regiones VII y X, que cuentan con proyectos de generación eléctrica, la inversión se concentra en las regiones metropolitana, VII y V, es decir, en los princi- 
pales centros demográficos y urbanos del país. Estas tres regiones suman $\mathbf{4 3 . 2 \%}$ de los montos proyectados en el área de servicios. Al agregar las regiones VII y X, se llega casi a los dos tercios del total.

En contraste con lo anterior, seis de las 13 regiones (I, II, IV, IX, XI, XII) sólo atraen un bajísimo 3.7\% de los recursos del sector de servicios. Casos particularmente opuestos resultan ser los de la II región ( $25.6 \%$ en bienes; $0.3 \%$ en servicios) y la IX región $(6.3 \%$ versus $0.4 \%$ respectivamente). Al contrario, destaca la VII región, con $0.7 \%$ en bienes y $8.4 \%$ en servicios. En esta misma situación están, en menor medida, las regiones $\mathrm{V}$ y metropolitana.

La región VIII es prácticamente la única que presenta alta captación de inversiones en ambos sectores. Un caso similar, aunque de menor equilibrio, es el de la X región. En cambio, la IV aparece desfavorecida en los dos casos.

De la comparación entre inversiones en el sector productor de bienes, por una parte, y de contribución regional a la producción de bienes y de bienes exportados por otra, se concluye que en general las inversiones se distribuyen más bien en proporción a las exportaciones de cada región. En otras palabras, la inversión en producción de bienes se relaciona más con el mercado externo que con el local.

En fin, si se considera que, con la excepción de la VIII región, las inversiones en los sectores productores de bienes y de servicios se orientan a distintas regiones, se verifica que los recursos de capital y el potencial de crecimiento asociado a ellos se distribuyen tendiendo a un cierto equilibrio interregional. Sólo habría dos excepciones: las regiones IV y VI, con inversiones mucho menos que proporcionales a su participación nacional. Ambas resultan perdedoras tanto en el sector productor de bienes como en el de servicios, más allá de las imperfecciones propias de la cartera de proyectos catastrados.

\section{Conclusiones}

El análisis expuesto permite sintetizar conclusiones referidas a la productividad de bienes, las exportaciones y las inversiones regionales.

Respecto de la primera variable, las cifras confirman fuertes cambios regionales entre 1970 y 1990. En efecto, todas las regiones -salvo el polo central constituido por la V y la metropolitanaincrementan su contribución al PGB nacional de bienes. En 1990, la región con mayor producción de bienes es la VI, y la de menor producción resulta la metropolitana. 
En materia de exportaciones regionales se verifica un patrón de dispersión relativa: cinco de las 13 regiones aportan tres cuartas partes del total nacional. En contraste, sólo dos regiones, la V y la metropolitana -las mismas que bajan su productividad de bienes- concentraron o al menos canalizaron más de $70 \%$ de las importaciones en 1990.

La II región hace la mayor contribución a las exportaciones del país. La menor corresponde a la IX. Las regiones menos "especializadas" en exportar son la IX y la RM: sólo 7.3\% del PGB de esta última se orienta al mercado externo. Las tres regiones sedes metropolitanas contribuyen con $63 \%$ del PGB y sólo $34 \%$ de las exportaciones.

Las inversiones -indicador que anticipa tendencias- divergen en su orientación territorial según se trate del sector productor de bienes o el de servicios. En cuanto al primero, tres regiones concentran más de la mitad de los valores proyectados. Las que corresponden al del segundo sector, en cambio, tienden a localizarse en las regiones sedes metropolitanas. El resultado de esta divergencia que denota una especialización regional, hace evidente la coexistencia de los efectos territoriales del nuevo modelo con los del antiguo patrón económico-espacial que, aunque desdibujado, acusa aún su enorme volumen e inercia.

En definitiva, la apertura económica y el desarrollo exportador, en el contexto más amplio de las políticas de liberalización y privatización, descentralización y modernización, han detonado un proceso de ajuste territorial, de carácter estructural, del que emergen nuevas regiones productoras de bienes, de exportación y domésticas.

Si bien el país ha avanzado sustantivamente como un todo, en la integración a los mercados internacionales y en la diversificación de su base económica y de exportación, las regiones muestran muy disímiles grados de apertura y una marcada especialización.

Con economías más o menos abiertas o cerradas, y orientadas unas al mercado doméstico y otras al sector externo, las regiones presentan dinámicas y perspectivas heterogéneas, a la vez que una muy desigual sensibilidad o vulnerabilidad frente a los fenómenos locales o mundiales, y también ante los impactos de las políticas macroeconómicas y de comercio exterior.

Si la especificidad de los sectores productores de bienes y de servicios permite una distinción analítica en términos económicos y territoriales, su interacción demanda una reflexión que en el campo regional enfrenta signos que se alejan de las controvertidas hipótesis de equilibrio y homogeneidad.

De hecho, mientras el PGB per cápita varía menos de seis veces entre las regiones, las inversiones totales por habitante lo ha- 
cen en 29 veces, y las exportaciones per cápita en más de 280 veces. Aquéllas y éstas actúan como factores de diferenciación, si no de discriminación más fuertes que el producto geográfico. $\mathrm{He}$ aquí un nuevo desafío para las políticas y el desarrollo regionales.

\section{Bibliografía}

Aninat, Eduardo (1990), "Investment opportunities in Chile: results from a survey of projects 1990-95", Seminario Oportunidades de Inversión en Chile, Santiago, Banco Central de Chile, Comité de Inversiones Extranjeras, Comisión de las Comunidades Europeas, marzo de 1990.

Banco Central de Chile (1983), Indicadores de Comercio Exterior, Dirección de Operaciones, Banco Central de Chile, diciembre.

(1991), Boletín mensual, núm. 760, Banco Central de Chile, junio.

Behrens, Roberto (1990). "La estrategia de desarrollo en Chile y el papel del capital extranjero: 1974-1989", Santiago Unidad Conjunta CEPALCET sobre Empresas Transnacionales.

Cuadra, Sergio de la (1988), "Necesidades de inversión en el sector exportador", en Estudios Monetarios, núm. X, Banco Central de Chile, septiembre.

Daher, Antonio (1990a), "Ajuste económico y ajuste territorial en Chile", en E. Laurelli y J. Lindenboim (comp.), Reestructuración Económica Global. Efectos y politicas territoriales, Buenos Aires, Ediciones Ceur, pp. 113-128.

- (1990b), "Políticas macroeconómicas, tipo de cambio y desarrollo territorial", en Revolución Tecnológica y Reestructuración Productiva: Impactos y Desafíos Territoriales, Buenos Aires, ILPES/ONU, IEUUC, Grupo Editor Latinoamericano, pp. 457-478.

Odeplan (1990a), "Estadísticas Regionales. Información macroeconómica”, Santiago, Oficina de Planificación Nacional.

—_ (1990b), "Estadísticas Regionales. Población e indicadores sociales", Santiago, Oficina de Planificación Nacional.

Servicio Nacional de Aduanas (1990), "Memoria 1990", Servicio Nacional de Aduanas, Chile. 